\title{
Mushroom's Evaluation Based on FT-IR Fingerprint and Chemometrics
}

\author{
Ioana Feher ${ }^{\mathrm{a}}$, Cornelia Veronica Floare-Avram ${ }^{\mathrm{a}}$, Florina-Dorina Covaciu ${ }^{\mathrm{a}}$, Olivian Marincas ${ }^{\mathrm{a}}$, \\ Romulus Puscas ${ }^{\mathrm{a}}$, Dana Alina Magdas ${ }^{\mathrm{a}}$, Costel Sârbu ${ }^{\mathrm{b}}$
}
${ }^{a}$ National Institute for Research and Development of Isotopic and Molecular Technologies, 67-103 Donat Street, 400293, Cluj-Napoca, Romania
${ }^{b}$ Babeș-Bolyai University, Faculty of Chemistry and Chemical Engineering, 11 Arany János, 400028, Cluj-Napoca, Romania

Corresponding author: veronica.avram@itim-cj.ro

\begin{abstract}
Edible mushrooms have been recognized as highly nutritional food for a long time, due to their specific flavor, texture and also for therapeutic effects. This study proposes a new simple approach, based on FT-IR analysis, followed by statistical methods, in order to differentiate three wild mushrooms species from Romanian spontaneous flora, namely Armillaria mellea, Boletus edulis and Cantharellus cibarius. The preliminary data treatment consisted of data set reduction with principal component analysis (PCA), which provided scores for the next methods. Linear discriminant analysis (LDA) manage to $100 \%$ classify the three species and the cross validation step of the method returned $97.4 \%$ of correctly classified samples. Only one A. mellea sample overlapped on B. edulis group. When $\mathrm{kNN}$ was used in the same manner as LDA, the overall percent of correctly classified samples from the training step was $86.21 \%$, while for holdout set the percent raised at $94.74 \%$. The lowered values obtained for the training set was due to one $C$. cibarius sample, two B. edulis and five A. mellea, which were placed to other species. Anyway, for holdout sample set, only one sample from B. edulis was misclassified. The fuzzy c-means clustering (FCM) analysis successfully classified investigated mushroom samples according to their species, meaning that in every partition the predominant specie had the biggest DOMs, while samples belonging to other specie had lower DOMs.
\end{abstract}

Keywords: mushrooms, FT-IR, chemometric, machine learning 


\section{Introduction}

Edible mushrooms have been recognized as highly nutritional food for a long time due to their specific flavor, texture and also for therapeutic effects. From the nutritional point of view, mushrooms represent an important source of proteins, fibers, minerals and also polyunsaturated fatty acids, their proportion having a large variation among different species. Regarding the vitamins content, its represent the only vegetarian source of vitamins D [1] and an important source of B group vitamin [2]. Moreover, mushrooms serve also as vegetarian source of protein [3]. On the other hand, wild mushrooms are thought to be richer in flavor, taste, texture, nutrition and medical effects [4].

Due to their beneficial effects upon human health, their demand is continuously growing and is expected to grow even more in the future. It is well known that, due to their soft texture, mushrooms have a short lifetime, around five days, and different types of post harvest procedures are usually applied in order to preserve as long as possible their availability [5]. There are three main classes of preservation procedures: thermal (drying/freezing), chemical (edible coatings, film, washing solutions) and physical (packing, irradiation, pulse electric field, ultrasound) [6]. Another reason for applying conservation steps in mushrooms preparation is the seasonal variability of some wild species. All these conservation methods also contribute to the preservation of their nutritional and nutraceutical value. Every procedure has advantages and drawbacks, for example, drying process, which is the first methods of choice [7], offers a more flavor taste of dried mushrooms, comparing to the fresh ones, but modify the content of bioactive compounds and nutrients [8].

In Romania, for the fifth last consecutive year, the market recorded an increase of the exported wild mushrooms quantities. The main destinations countries were Italy, followed by Hungary and Spain. China is the main producer of cultivated, edible mushrooms. Collecting wild edible mushrooms for consumption is widely practiced in many countries, including Romania [9, 10]. The consumption of mushrooms is expected to increase, since consumers are becoming aware of the helpful benefits brought in the diet [11].

Among all available analytical techniques able to evaluate different types of compounds in food matrices, such nuclear magnetic resonance (NMR), high performance liquid chromatography (HPLC) [12] Fourier-transform infrared spectroscopy (FT-IR) is one of the most widely used method to identify chemical compounds and elucidate chemical structure, 
having as main advantages the rapid, reagent less and high-throughput operation, within a wide range of matrices [13]. It allows rapid and simultaneous characterization of different functional groups, such as lipids, proteins, and polysaccharides [14]. For food quality and control field, FTIR spectroscopy is an important tool, due to low operating costs and good performance [15]. The record FT-IR spectra represents a global assessment of a specific matrix, more precisely a molecular fingerprint, which are very suitable for characterization, differentiation or identification of different matrices, including mushrooms [16].

The aim of the present study was the differentiation of three investigated mushrooms species (Armillaria mellea, Boletus edulis and Cantharellus cibarius) through the development of a differentiation tool, made up of a fast and efficient analytical technique coupled with different chemometric methods.

\section{Materials and methods}

\subsection{Sample collection}

For fulfilling the aim of this study, a number of 77 wild-grown mushrooms samples, belonging to three different species, namely Armillaria mellea, Boletus edulis and Cantharellus cibarius, were collected and analyzed. The samples were collected during summer, in 2019, from different geographical area located mainly near Cluj County, Romania. The distribution of samples according to their species was as follows: Armillaria mellea, 12 samples, Boletus edulis 31 samples and Cantharellus cibarius, 34 samples.

\subsection{Sample preparation and analysis}

In the laboratory, the samples were dried in an oven at $60^{\circ} \mathrm{C}$, until constant weight. Subsequently, the dried samples were grounded into a fine powder and store at $4^{\circ} \mathrm{C}$ for further analysis. The powder of each sample was mixed uniformly with $\mathrm{KBr}$ ad then pressed into a tablet using a tablet press.

The FT-IR spectrometer (PerkinElmer, USA) used to perform the mushrooms analysis was equipped with a thermal deuterated triglycine sulfate (DTGS) detector. The spectral range was $4000-400 \mathrm{~cm}^{-1}$ with resolution of $4 \mathrm{~cm}^{-1}$. For each sample the spectrum consisted of 64 scans, which were performed intriplicate and averaged. After recording the spectra, and prior other chemometric prelucration, all spectra were smoothed by Savitzky-Golay algorithms and 
linear baseline corrected. The spectra were further imported in OriginPro 2017 (OriginLab, Northampton, USA) and subjected to $[0,1]$ normalization.

\subsection{Chemometrics methods}

All chemometric methods were made using SPSS Statistics version 24 (IBM, USA) software. The first method which was applied to normalized spectra was principal component analysis (PCA). This method is one of the most used unsupervised pattern techniques, which is able to provide the reduction of large data set to smaller components called principal components (PC) or factors, with a minimum loss of original information. The obtained PCs are uncorrelated and appear in decreasing order of importance, an important aspect being the eigenvalues, which are a measure of components significance to the data set variance. Usually, the first two or three components retain a high percent of data variance.

A widely employed supervised chemometric methods used for classification purposes is linear discriminant analysis (LDA). Being a supervised method, a new variable must be created and every sample receives a code, corresponding to different discrimination criterion. LDA will find linear combinations of variables, called discriminant functions (DFs), creating a predictive model. While constructing the model, the method tries to maximize the distance among classes and to minimize the distance within the same class, thus providing a robust classification model, which consists only of representative features. A validation step is also made, using "leave-oneout cross validation", which implies the testing of each sample as a new one, using a model obtained without that sample [17]. The model performances are evaluated through the percent of correctly classified samples, a higher percent suggesting a stronger model. In this specific case, the LDA was applied for discovering the specific FT-IR bands, which can discriminate the three investigated mushrooms species.

Apart from LDA, another widely used classification method is represented by $\mathrm{k}$ nearest neighbor $(\mathrm{kNN})$, which is one of the simplest machine learning algorithms. This method is based on similarities between new samples and available data and puts the new sample within category that is most similar. An important aspect of this algorithm is that it does not need training, finds the neighbors nearest to the sample and divides them into categories. Thus, $\mathrm{kNN}$ is suitable for multivariate classification and has high classification accuracy when the category boundary is obvious [18]. 
Clustering is an unsupervised machine learning technique that implies the grouping of samples into different clusters and sample from the same cluster has high degree of similarity, while samples from different clusters have low degree of similarity. In fuzzy clustering, each point (sample) has a probability of belonging to each cluster, rather than completely belonging to just one cluster, as it is the case in the traditional k-means. Clustering and classification methods are useful for big data visualization, due to the fact that allow meaningful generalizations to be made by recognizing general patterns among them [19, 20]. In Fuzzy-C Means clustering, each point has a weighting associated with a particular cluster, so a point doesn't lie "in a cluster" as long as the association to the cluster is weak. The fuzzy C-means (FCM) algorithm, a method of fuzzy clustering, is an efficient algorithm for extracting rules and mining data from a dataset in which the fuzzy properties are highly common [21, 22].

\section{Results and discussion}

\subsection{FT-IR initial spectra of mushroom samples}

As it was previously mentioned, 77 wild-grown mushrooms samples, belonging to three different species, namely Armillaria mellea, Boletus edulis and Cantharellus cibarius were analyzed. The experimental spectra are presented in Figure 1.

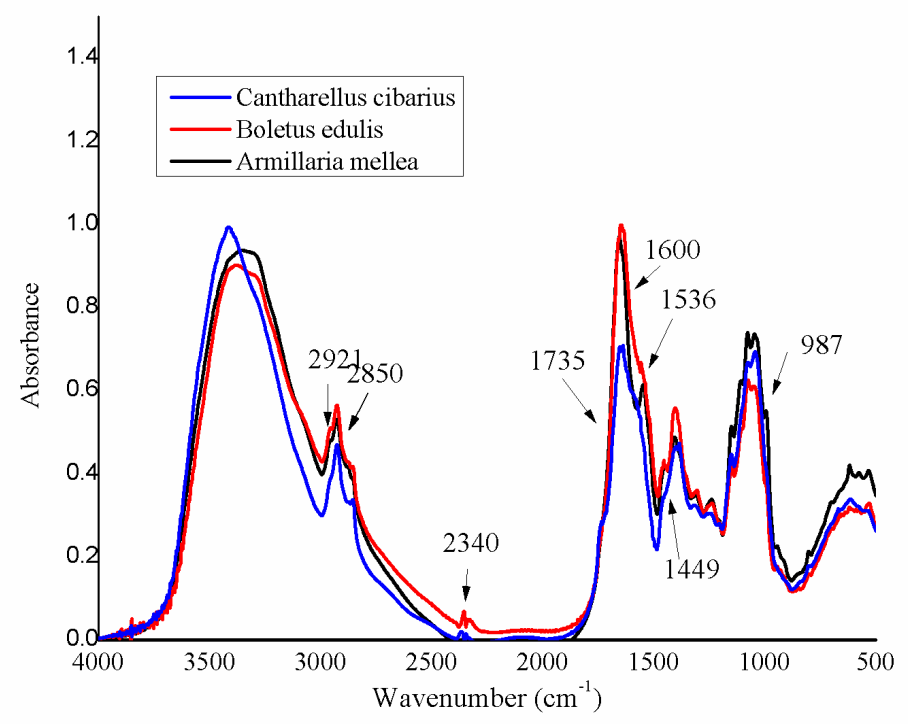

Figure 1. FT-IR spectra of three selected species 
At the first visual inspection of mushrooms samples, the most relevant differences in the spectra seem to be situated around the bands from: $2921 \mathrm{~cm}^{-1}, 2340 \mathrm{~cm}^{-1}, 1735 \mathrm{~cm}^{-1}, 1600 \mathrm{~cm}^{-1}$ $1546 \mathrm{~cm}^{-1}, 1433 \mathrm{~cm}^{-1}$ and $987 \mathrm{~cm}^{-1}$. According to literature, the organic compounds that are responsible for these differences are: saturated aliphatic esters $\left(1750,1733,1710 \mathrm{~cm}^{-1}\right)$, melanin $\left(1600 \mathrm{~cm}^{-1}\right)$, amide II group, maily chitosan $\left(1582,1550,1536 \mathrm{~cm}^{-1}\right)$, polysaccharides (1450$1425,1415 \mathrm{~cm}^{-1}$ ) [23]. Peaks in the region $1000-400 \mathrm{~cm}^{-1}$ mainly belong to polysaccharides, such $\beta$-D-glucans and the pyranose form of glucose [24].

The impossibility to identify all spectral differences among the analyzed species is not surprising for spectroscopic analysis of complex matrices, therefor different chemometric methods are required in order to give a better and more comprehensive characterization of matrices.

\subsection{Chemometric processing}

For chemometric dara processing only the fingerprint region $1800-400 \mathrm{~cm}^{-1}$ was took into account. Even so, due to the large dimension of obtained FT-IR matrix, which is very difficult to be further chemometrically processed, first a factorial analysis for dimensions reduction was applied, namely PCA. In this case, the PCA analysis was run using the following key parameters: extraction method, principal components, rotation methods, Varimax with Kaiser Normalization. An impressive number of PCs was obtained, but only PC with eigenvalue higher that one was retained fur further analysis. Usually the first PCs obtained explain the largest percent of data variation. In this case the first fourteen PCs have eigenvalues higher than one and explained a cumulative variance of $99.53 \%$, being representative for next chemometric treatment.

For discrimination of the three investigated mushrooms species a new variable was created, and each sample received a code corresponding to their species, as follows: code 1 for Cantharellus cibarius, code 2 for Boletus edulis and code 3 for Armillaria mellea. This variable was used as grouping variables, while the PCs obtained from previously were employed as independent variables. Wilks lambda was chosen as discrimination method. The percent obtained for initial classification was $100 \%$, and the cross validation step of the method returned $97.4 \%$ of correctly classified samples. From the classification table it could be observed that in the cross validation procedure only one sample of Armillaria mellea was assigned to Boletus edulis group. The graphical representation is presented in Figure 2. 


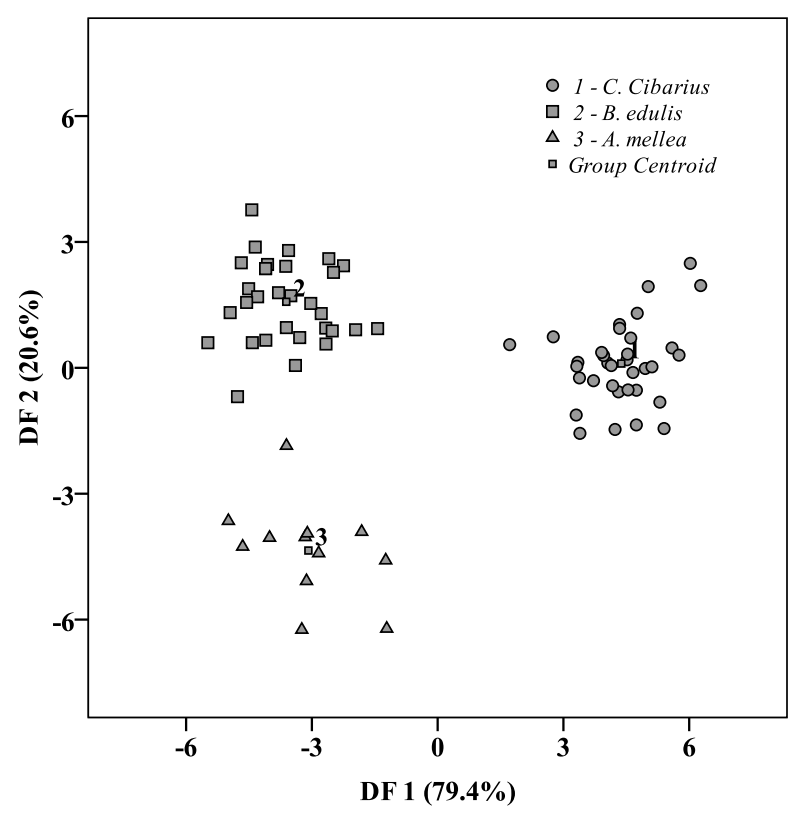

Figure 2. Mushrooms differentiation after applying LDA

Since three groups were compared, two discriminant functions were obtained. These functions were statistically significant $(\mathrm{p}=0.001)$ and Wilks values were 0.012 and 0.195 , respectively. The first function ( $\mathrm{DF} 1,79.4 \%)$ contained the largest values for the majority of PCs, the second function (DF2, 20.6\%) was given by only two PCs. Generally, the largest values (loadings) of each point from the spectra suggest a higher contribution of that variable to corresponding PC, thus only values higher than 0.5 were considered. In this case, by inspecting the rotated component matrix obtained after running PCA, it could be observed that some parts of spectra were highlighted as being different among investigated mushrooms species. To the first PC the corresponding part of spectra is from 400 to $925 \mathrm{~cm}^{-1}$. According to paper published by Meenu et al. the region below $900 \mathrm{~cm}^{-1}$, this region could be assigned to $\alpha$-glucans and $\beta$ glucans [25]. These compounds belong to polysaccharides groups and the most common glucans from fungi are $\beta$-glucans [26], whose beneficial effect upon human health is well known, immunomodulatory, antitumoral, hipolipidemic and antimicrobial [27]. Glucans are responsible for a proper functioning and health of cells from wall structure. Other significant areas from spectra retained by the second PC are those from $999-1121 \mathrm{~cm}^{-1}$ and $1141-1155 \mathrm{~cm}^{-1}$. These two regions from spectra could serve as a valuable indicator of mushrooms genius, although particular species cannot be identified through spectroscopic techniques [28, 29]. The next PC grouped another two spectral regions, $1484-1559 \mathrm{~cm}^{-1}$ and $1598-1695 \mathrm{~cm}^{-1}$. The next two PC 
grouped another significant region of FT-IR spectra, namely: $1715-1800 \mathrm{~cm}^{-1}$ and $1548-1561$ $\mathrm{cm}^{-1}$.

Taken into consideration that some of the last obtained PCs did not contain any specific spectra regions and also that no specific points were identified, a more powerful classification method was employed, this time having as variables the entire FT-IR spectra.

Among the machine learning algorithms, $\mathrm{k}$ nearest neighbor is the most simple and accessible one. In this case, $\mathrm{kNN}$ was applied for highlighting the features used to predict a certain mushrooms species. As target variable for the model the species variable was set, having specific code for each sample. The specific number of neighbors was set at five, while the distance among identified neighbors was measured through Euclidian distance. Also a features selection was adopted and a weight by importance of each point was selected [30, 31]. The partition of sample between training and holdout sets was randomly assigned, having a proportion of $70 \%$ and $30 \%$, respectively (Table 1). The classification table, obtained after running $\mathrm{kNN}$, is presented below.

Table 1. Classification table of mushrooms samples, obtained after kNN modeling

\section{Predicted}

Partition
C. cibarius
B. edulis
A. mellea

Percent correct

\begin{tabular}{cccccc}
\hline \multirow{4}{*}{ Training } & C. cibarius & 27 & 1 & 0 & $96.43 \%$ \\
& B. edulis & 1 & 18 & 1 & $90.00 \%$ \\
& A. mellea & 2 & 3 & 5 & $50.00 \%$ \\
& Overall percent & $51.73 \%$ & $37.94 \%$ & $10.35 \%$ & $86.21 \%$ \\
\hline \multirow{3}{*}{ Holdout } & C. cibarius & 6 & 0 & 0 & $100 \%$ \\
& B. edulis & 0 & 10 & 1 & $90.91 \%$ \\
& A. mellea & 0 & 0 & 2 & $100 \%$ \\
& Overall percent & $31.58 \%$ & $52.64 \%$ & $15.79 \%$ & $94.74 \%$ \\
\hline
\end{tabular}

In the training step, the overall percent of correctly classified samples is $86.21 \%$, while for holdout set the percent raise at $94.74 \%$. The lowered values obtained for the training set is due to one $C$ cibarius sample, two B. edulis and five A. mellea, which were placed to other 
species. Anyway, for holdout sample only one sample from B. edulis was misclassified. Regarding the features selection, only three points were selected: $1746 \mathrm{~cm}^{-1}, 1510 \mathrm{~cm}^{-1}$ and 1388 $\mathrm{cm}^{-1}$. The samples distribution between the two set, according to selected features is presented in Figure 3, below:

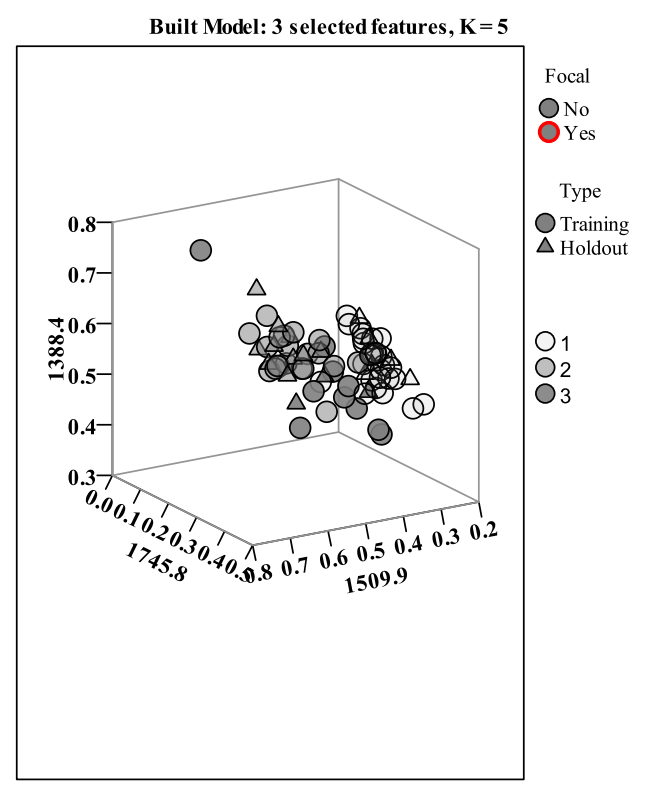

Figure 3. kNN modeling of mushrooms samples, with three features selected and five neighbors.

It should be noticed that the results obtained using PCA-LDA and kNN are very similar, in terms of prediction accuracy. Regarding the obtained predictors, it should be mentioned that except $1746 \mathrm{~cm}^{-1}$, which appeared also in LDA classification, the other two bands are new predictors. This could lead to the conclusion that these two approaches are complementary.

The number of groups for fuzzy c-means clustering (FCM) analysis was chosen according to the three investigated species, namely 3. FCM produced 3 fuzzy partitions, which were all represented by a prototype (a cluster center with the spectrum corresponding to the fuzzy robust means of the original IR spectra characteristics for 77 samples weighted by degree of membership (DOMs) corresponding to each partition. To compare the partitions, the similarities and differences among samples, both the spectra of the prototypes corresponding to the three fuzzy partitions (A1-A3) obtained by applying FCM and DOMs of samples corresponding to all fuzzy partitions have to be analyzed. The results presented in Table 2 and 
Figure 4 clearly illustrates the most specific characteristics of each fuzzy partition and their (dis)similarity and the samples assigned according to their DOMs.

The fuzzy partition A1, for example, includes almost all samples from 13-43 group (Boletus edulis) and also samples 1, 10 from A2 and 70, 75 from A3 with relatively small DOMs.Th remaining samples belonging to this group $(14,24,28)$ were included in A2 partition, with small DOMs, except sample 28 (0.7454), while samples 25, 29, 31, 35, 41 were placed in A3 partition with moderate DOMs

Except the samples 14, 24 and 28 from Boletus edulis, the second fuzzy partition A2 contains the majority of samples from 1-12 (Armillaria melea) and also samples 44, 48, 49, 50, 58, 61, 62, 63, 66, 68, 72, 73 from Chantharellus cibarius group, with relatively high and moderate DOMs.

On the contrary, the third fuzzy partition A3 includes the majority of samples from 44-77 belonging to Cantharellus cibarius and also samples 3 and 5 from A2 and 25, 29, 31, 35, 41 from A1 with small DOMs except 29 (0.7029).

Table 2. The three fuzzy partitions obtained by applying fuzzy c-means clustering method

\begin{tabular}{|c|c|c|}
\hline $\begin{array}{l}\text { Fuzzy } \\
\text { partition }\end{array}$ & $\begin{array}{c}\text { Samples of each partition ranking in } \\
\text { decreasing order of DOMs }\end{array}$ & $\begin{array}{l}\text { DOMs } \\
\text { range of samples }\end{array}$ \\
\hline A & $1, \ldots, 77$ & - \\
\hline A1 & $\begin{array}{c}110 \\
131516171819202122232627303233343637 \\
38394042437075\end{array}$ & $0.9789-0.4021$ \\
\hline $\mathrm{A} 2$ & $\begin{array}{c}246789111214242844484950586162 \\
6366687273\end{array}$ & $0.9224-0.4471$ \\
\hline A3 & $\begin{array}{c}3525293135414546475152535455 \\
565759606465676971747677\end{array}$ & $0.9147-0.3876$ \\
\hline
\end{tabular}




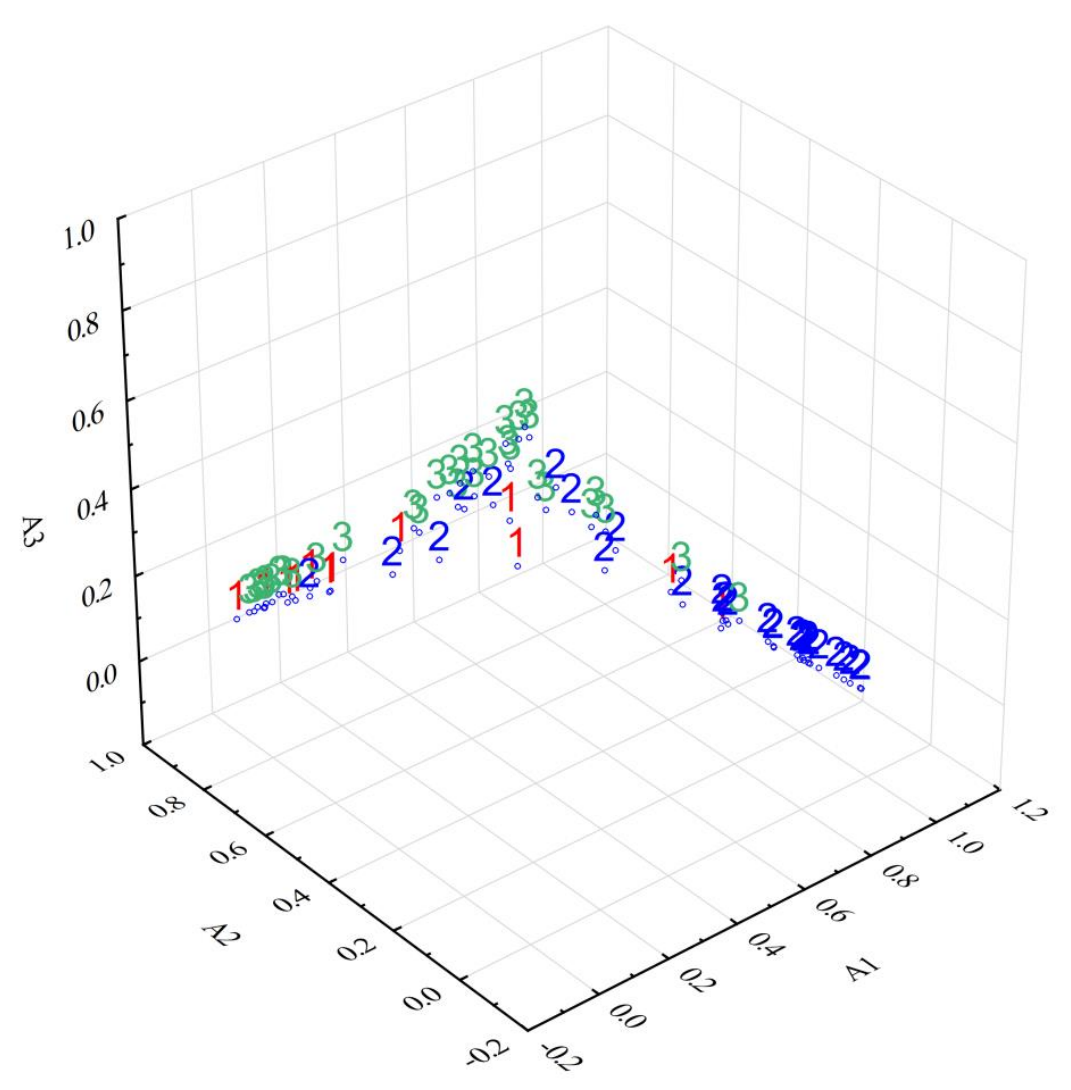

Figure 4. 3-D scatterplot of DOMs corresponding to partition A1, A2 and A3

The fuzzy c-means clustering analysis successfully classified investigated samples according to their species, meaning that in every partition the predominant specie had the biggest DOMs, while samples belonging to other specie had lower DOMs.

\section{Conclusions}

The present study demonstrated the great potential of combined analytical techniques, such FT-IR and different chemometric processing methods. By applying LDA on scores obtained after PCA, the obtained percent for initial classification was $100 \%$, and the cross validation step of the method returned $97.4 \%$ of correctly classified samples. Only one A. mellea sample overlapped on B. edulis group. When $\mathrm{kNN}$ was used in the same manner as LDA, the overall percent of correctly classified samples from the training step was $86.21 \%$, while for holdout set the percent raised at $94.74 \%$. The lowered values obtained for the training set was due to one $C$. cibarius sample, two B. edulis and five A. mellea, which were placed to other 
species. Anyway, for holdout sample set, only one sample from B. edulis was misclassified. These two statistical techniques proved to be complementary, only one common band being found $\left(1746 \mathrm{~cm}^{-1}\right)$. The FCM analysis successfully classified investigated mushroom samples according to their species, meaning that in every partition the predominant specie had the biggest DOMs, while samples belonging to other specie had lower DOMs.

Author contributions: conceptualization, methodology and writing, I.F.; D.A.M.; sample collection and analytic measurements C.F.; R.P.; V.F.A.; O.M.; software prelucration I.F.; C.S.; writing I.F.; V.F.A.; C.F.; O.M.; revision D.A.M.; C.S. All authors have read and agreed to the published version of the manuscript.

Funding: This work was supported by Ministry of Research, Innovation and Digitalization, grant number PD 90/2020, within PNCDI III.

Institutional Review Board Statement: Not applicable.

Informed Consent Statement: Not applicable

Conflict of interest: The authors declare no conflict of interest. 


\section{References}

1. Cardwell, G.; Bornman, J.F.; James, A.P.; Black, L.J. A review of mushrooms as a potential source of dietary vitamin D. Nutrients. 2018, 10, 1498-1509

2. Rathore, H. Prasad; S.; Sharma, S. Mushroom nutraceuticals for improved nutrition and better human health: A review. Pharma Nutr. 2017, 5(2), 35-46.

3. Das, B.; Rajkonwar, J.; Jagannath, A.; Raul, P.K.; Deb, U. Infra-red spectra of different species of cultivated oyster mushrooms: Possible tool for identifying bioactive compounds and establishing taxonomic linkage. Def Life Sci J. 2020, 5, 118-124.

4. Li, J.; Wu, H.; Wang, L.; Huang, Y.; Wang, L. Key taste components in two edible Boletus mushrooms using widely targeted metabolomics. Biochem Syst Ecol. 2021, 96, 104268.

5. Granato, D.; Putnik, P.; Kovacevic, D.B.; Santos, J.S.; Calado, V.; Rocha, R.S.; Gomes Da Cruz, A.; Jarvis, B.; Rodionova, O.Y.; Pomerantsev, A. Trends in Chemometrics: Food Authentication, Microbiology and Effects of Processing. Compr Rev Food Sci Food Saf. 2018, $17,663-677$

6. Marçal, S.; Sousa, A.S.; Taofiq, O.; Antunes, F.; Morais, A.M.M.B.; Freitas, A.C.; Barros, L.; Ferreira, I.C.F.R.; Pintado, M. Impact of postharvest preservation methods on nutritional value and bioactive properties of mushrooms. Trends Food Sci Technol. 2021, 110, 418-431.

7 .Zhang, K.; Pu, Y.Y.; Sun, D.W. Recent advances in quality preservation of pastharvest mushrooms (Agaricus bisporus): A review. Trends Food Sci Technol. 2018, 78, 72-82.

8. Yadav, D.; Negi, P.H. Bioactive components of mushrooms: Processing effects and health benefits. Food Res Int. 2021, 148, 110599

9. Mleczek, M.; Siwulski, M.; Budka, A.; Mleczek, P.; Budzyńska, S.; Szostek, M.; Kuczyńska-

Kippen, N.; Kalač, P.; Niedzielski, P.; Gąsecka, M.; Goliński, P.; Magdziak, Z.; Rzymski, P. Toxicoligical risks and nutritional value of wild edible mushrooms species - a half century monitoring study. Chemosphere. 2021, 263, 128095

10. Zsigmond, A.R.; Varga, K.; Harangi, S.; Baranyai, E.; Urak, I. Elemental profile of edible mushrooms from a forest near a major Romanian city. Acta Univ Sapientiae Agric Environ. 2015, 7, 98-107.

11. Roise, D.J.; Baars, J.; Tan, Q. Current overview of mushrooms production in the world, Chapter in book Edible and medicinal mushrooms. Technology and Applications, ed. Zied, Diego Cunha, Pardo-Gimenez Arthuro, Wiley-Blackwell, 2017. 
12. Gong, P.; Wang, S.; Liu, M.; Chen, F.; Yang, W.; Chang, X.; Liu, N.; Zhao, Y.; Wang, J.; Chern, X. Extraction methods, chemical characterizations and biological activities of mushrooms polysaccharides: A mini-review. Carbohydr Res. 2020, 494, 108037

13. Bebu, A.; Andronie, L.; Maties, A., Micle, S.; Darjan, S.; Culcear, O. Comparative analysis between mushrooms Lactarius Piperatus and Agaricus Bisporus (Champignon) using FT-IR Spectroscopy. Sci Papers, Series B, Hortic. 2020, vol LXIV, No 1, 633-637.

14. Zavastin, D.E.; Biliută, G.; Dodi, G.; Macsim, A.; Lisa, G.; Gherman, S.P.; Breabăn, I.G.; Miron, A.; Coseri, S. Metal content and crude polysaccharide characterization of selected mushrooms growing in Romania, J Food Comp Anal. 2018, 67, 149-158.

15. O'Gorman, A. Use of Fourier Transform Infrared Spectroscopy and Chemometric Data Analysis To Evaluate Damage and Age in Mushrooms (Agaricus bisporus) Grown in Ireland. $J$ Agr Food Chem. 2010, 58 (13), 7770-7776.

16. D'Souza, R.A.; Kamat, N.M. Potential of FT-IR spectroscopy in chemical characterization of Termitomyces Pellets. J Appl Biol Biotechnol. 2017, 5, 080-084.

17. Liu, H.I.; Zang, Y.; Zhao, X.; Tong, H. Improved geographical origin discrimination for tea using ICP-MS and ICP-OES techniques in combination with chemometric approach. $J$ Sci Food Agr. 2020, 100, 3507-3516.

18. Gao, R.; Chen, C.; Wang, H.; Chen, C.; Yan, Z.; Han, H.; Chen, F.; Wu, Y.; Wang, Z.; Zhou, Y.; Si, R.; Lv, X. Classification of multicategory edible fungi based on the infrared spectra of caps and stalks. PLoS One. 2020, 15(8): e0238149.

19. Sârbu, C.; Pop, H.F. Fuzzy Soft-Computing Methods and Their Applications in Chemistry. Rev. Comput. Chem. 2004, 20, 249-253.

20. Feher, I.; Magdas, D.A.; Voica, C.; Cristea, G.; Sârbu, C. Fuzzy Divisive Hierarchical Associative-Clustering Applied to Different Varieties of White Wines According to Their MultiElemental Profiles. Molecules. 2020, 25, 4955-4965.

21. Ruspini, E.H.; Bezdek, J.C.; Keller, J.M.; Fuzzy Clustering: A historical perspective. IEEE Comput Intell Mag. 2019, 14, 45-55.

22. Khang, T.D.; Vuong, N.D.; Tran, M.K.; Fowler, M. Fuzzy C-means clustering algoritm with multiple fuzzification algorithms. Algorithms. 2020, 13, 158-169.

23. Bekiaris, G.; Koutrotsios, G.; Tarantilis, P.A.; Pappas, C.S.; Zervakis, G.I. FT-IR assessment of compositional changes in lignocellulosic wastes during cultivation of Cyclocybecylindracea 
mushrooms and use of chemometric models to predict production performance. J Mater Cycles Waste Manag. 2020, 22, 1027-1035.

24. Yao, S.; Li, T.; Li, J.Q.; Liu, H.G.; Wang, Y.Z. Geographic identification of Boletus mushrooms by data fusion of FT-IR and UV spectroscopies combined with multivariate statistical analysis. Spectrochim Acta. A Mol Biomol Spectrosc. 2018, 198, 257-263

25. Meenu, M.; Xu, B. Application of vibrational spectroscopy fro classification, authentication and quality analysis of mushrooms: A concise review. Food Chem. 2019, 289, 545-557.

26. Morales, D.; Rutckeviski, R.; Villalva, M.; Abreu, H.; Soler-Rivas, C.; Santoyo, S.; Iacomini, M.; Ribeiro Smiderle, F. Isolation and comparison of $\alpha$ - and $\beta$-D-glucans from shiitake mushrooms (Lentinula edodes) with different biological activities. Carbohydr Polym. (2020), 229, 115521

27. Khan, A.A.; Gani, A.; Masoodi, F.A.; Mushtaq, U.; Naik, A.S. Structural, rheological, antioxidant, and functional properties of $\beta$-glucan extracted from edible mushrooms Agaricusbisporus, Pleurotusostreatus and Coprinus attrimentarius. Bioact Carbohydr Diet Fibre. 2017, 11, 67-74.

28. Mohacek-Grocev, V.; Bozac, R.; Puppels, G.J. Vibrational spectroscopic characterization of wild growing mushrooms and toadstools. Spectrochim Acta. A Mol Biomol Spectrosc. 2001, 57(14), 2815-2829

29. Ruthes, A.C.; Cantu-Jungles, T.M; Cordeiro, L.M.C.; Iacomini, M. Prebiotic potential of mushrooms D-glucans: implications of physicochemical properties and structural features. Carbohydr Polym. 2021, 262, 117940.

30. Dhanabal, S.; Chandramathi, S. A review of various k-nearest neighbor query processing techniques. Int J Comput Appl. 2011, 31(7), 14-22.

31. Gilani, S.J.; Imam, S.S.; Ahmad, J. Application of fingerprinting techniques in authentication and traceability of fruits and vegetables. in Fingerprinting techniques in Food Authentication and Traceability, $1^{\text {st }}$ ed.; Siddiqi, K.S.; Nollet, L.M.L., CRC Press, Taylor and Francis Group, UK, 2019, pp. 341-342. 